trolled by anticonvulsants.

Of these 23 cases, 15 cases were followed up for more than 3 years. The results are as follows: of 10 cases of corticogenic focal epilepsy 2 cured, 3 excellent, 4 improved and 1 not changed.

The results appear to be very good. The streotaxic putamectomy was also performed bilaterally for all centrencephalic type of convulsion. The selective stereotaxic putamectomy for the anterior part of putamen was performed for hemiconvulsive and partial convulsive type.

In 5 cases of centrecephalic generalized epilepsy, there were 2 excellent cases, 2 improved and 1 not changed cases.

These operations for the centrencephalic type did not give a satisfactory result as to be able to stop anticonvulsant but able to reduce the amount of anticonvulsants to satisfactory level.

The stereotaxic putamectomy appears to play a significant role to block the reverbrating circuit of seizure discharge in the centrencephalic epilepsy.

The conclusion: the stereotaxic putamectomy has been found to be effective in the treatment of the corticogenic focal epilepsy under the strict indication for operation .

\title{
17. Indications of Forel-H-tomy for the Treatment of Epilepsy
}

\author{
Dennosuke Jinnai, Heitaro Mogami, Kiyoo KamiKawa, Jiro MuKawa, \\ Takuya IKEDA and Kiyoshi KobaYASHI \\ Department of Neurosurgery, Kyoto University Medical School
}

1) This sterotaxic destruction of the Forel's $H$ field has been attempted for the treatment of intractable epileptic patients both of idiopathic and symptomatic geneses.

2) They are composed of 11 for each male and female. Age was 7 to 33 years at the period of surgery. The follow-up period is 6 to 56 months.

3) The target is $4 \mathrm{~mm}$ ventral to the CA-CP line, $2 \mathrm{~mm}$ posterior to the midpoint and $8 \mathrm{~mm}$ lateral to the midsagittal line. The cryosurgical lesion was $6 \mathrm{~mm}$ diameter. Bilateral procedure was scheduled in 2 weeks in interval.

4) The ipsilateral complete lesion resulted all in excellent in cases of focal or hemispheric abnormal types of symptomatic epilepsy: 3 out of 5 unilateral and 2 out of 9 bilateral procedures. The bilateral complete lesions resulted all in excellent in cases of idiopathic epilepsy: 4 out of 8 bilateral procedures.

5) These data give an evidence of necessity of making complete lesion at the ipsilateral Forel's $\mathrm{H}$ field on the abnormal hemisphere for the satisfactory result. The relation between the lesion of the Forel's $\mathbf{H}$ field and surgical result is summarized in the Table. 
6) Summarizing the experimental data, Forel-H-tomy inhibits the Metrazolinduced gran dmal and the electrically induced motor-cortical after-discharge, but not the limbic after-discharge. These correspond well with the clinical data.

\begin{tabular}{l|c|c|c|c}
\hline R-lesion & excellent & good & poor or no & total \\
\hline complete, both sides & 6 & 0 & 0 & 6 \\
$\begin{array}{l}\text { complete, one side incomplete, } \\
\text { other side }\end{array}$ & 0 & 1 & 1 & 2 \\
$\begin{array}{l}\text { incomplete, both sides } \\
\text { incomplete, one side out of } \mathrm{H},\end{array}$ & 0 & 4 & 1 & 5 \\
$\quad \begin{array}{c}\text { other side } \\
\text { total }\end{array}$ & 6 & 6 & 5 & 4 \\
\hline
\end{tabular}

\title{
18. Clinical Indication of Thalamic Pulvinatomy for Intractable Pain
}

\author{
Tatsuyuki Kudo, Nobuo Yoshir, Kimihiro Mizukami, \\ Tohoru Mine and Kazuma ADACHI \\ Division of Neurological Surgery, School of Medicine, Keio University \\ Shiro Shimizu, Hiroshi NaKahama, Sadao Aikawa and \\ Shinko NisHIOKA \\ Seishin Igaku Institute
}

There are some difference between the effect of sterotaxic operations with various invasion areas for intractable pain. We have performed thalamic pulvinotomy stereotaxically in 17 cases and have obtained excellent to good result. Out of these 17 patients, 7 suffered from postcerebrovascular accident, 8 from infiltration and metastasis of cancer, 2 from facial pain.

Subjective complaints of the pain, objective sensory change and the required medication to relieve pain are important as index of the pain. However, the feeling and expression of the pain are various individual patient. Therefore it seems to be important to asses the degree of patients' pain by recording their analgesic requirements before the surgical procedures. In cases which supposed to be under the inappropriate medication for pain relief, we give placebo or mild analgesics such as pyrabital at first. Then we can find appropriate kind and adequate dossage of medicaments to relieve pain. In 10 cases the injection of the narcotic was required to relieve pain, in 7 cases the injection of such drug combination as "phnobarbital and pyrabital" or "phenobarbital and tranquilizer" was required to relieve pain. Within 3 weeks after the thalamic pulvinotomy complete relief of the pain was obtained in 8 cases which was achieved in 10 